\title{
Transport: Referees 2018
}

The following is a list of referees who have reviewed papers for Transport between 1 January 2018 and 31 December 2018. The Institution of Civil Engineers is very grateful for their assistance.

We are continually looking for suitable reviewers for papers submitted to Transport. Papers published in the Proceedings of the ICE must be submitted to at least two independent referees to judge accuracy, style, impact, importance and interest.

If you are interested in reviewing articles on any topic related to transport, please submit your name, qualifications or CV, and areas of

Vahid Abolhasannejad

Ahmad Abu Abdo

Olufikayo Aderinlewo

Anwaar Ahmed

Huseyin Akbulut

Mir Ali

Mazen Al-Kheetan

Raul Almeida

Borja Alonso

Imad Al-Qadi

Jiri Ambros

Costas Anagnostopoulos

António Andrade

Alex Apeagyei

Justice Appiah

Stephen Arhin

Robert Armitage

John Armstrong

Ahmet Atalay

Richard Atta Boateng

Nii Attoh-Okine

Metin Aydin

Atef Badr

Manjurali Balya

Ozgur Baskan

Shy Bassan

Osman Bayrak

William Bergeson

Niyazi Özgür Bezgin

Justin Bishop

Ian Blackwell

Giovanna Borille

Simon Bowers

\section{David Brill}

Richard Brown

John Cairns

Mengqiu Cao

Carlos Carrion

Guillermo Castilla

Halim Ceylan

Muhammed Yasin Codur

Seosamh Costello

Brian Counter

Andre Dantas

Alessandro Di Graziano

Nancy Dutta

Morteza Esmaeili

Harry Evdorides

Han Fei

Aoife Foley

William Frith

Matthew Frost

Mohammad Ganji

Marinella Giunta

Sarath Chandra Gorthy

James Grenfell

Marco Guerrieri

Ismail Bulent Gundogdu

Ankit Gupta

Derek Halden

Wei-Ming Ho

John Hodgson

Bing Huang

Yue Huang

Zahangir Hussain

Timothy Hutchinson

expertise. We are in need of individuals who will agree to review papers in a timely fashion (within 3 to 4 weeks of receipt) and provide confidential feedback to the Editorial Advisory Panel concerning the quality of the paper and any suggested revisions that would be appropriate.

If you are such a person, please contact Kirsten Buchanan (tel.: +44 (0)207 665 2204; e-mail: kirsten.buchanan@ice.org.uk) for more information on the referee process.

Morteza Jalili Qazizadeh

Pierre Jouniaux

Sakdirat Kaewunruen

Misbah Khan

Murali Krishnan

Didin Kusdian

Giovanni Leonardi

Jan-Mou Li

Jun Liu

Maria Eugenia Lopez-Lambas

Tao Ma

lain MacGregor

Hooman Mansoori

Luca Mantecchini

Arash Mazaheri

Michael McNerney

Alejandra Medina

Miomir Miljković

Lo Siu Ming

Reza Mohammad Hasany

Haneen Mohammed

Carlos Moran Toledo

Matt Muirhead

Bhaven Naik

Pooya Najaf

Graham Newson

Chayut Ngamkhanong

James Nicholls

Mohd Osman

Bhoj Pantha

Grigorios Papageorgiou

Manoranjan Parida

John Parkin
Naga Pasupuleti

Dalibor Pesic

Basil Psarianos

Behzad Rahimzadeh

Amir Rassafi

Rajat Rastogi

John Rolt

Krishna Satti

Mohammad Shokrolah Shirazi

Oliver Shyr

Jaroslav Smutny

Ioanna Spyropoulou

Emily Tan

Yuksel Tasdemir

Tyrone Toole

Valeria Vignali

Weijie Wang

Mohamed Wehbi

Peter White

Iswandaru Widyatmoko

Shaopeng Wu

Baozhen Yao

Yok Hoe Yap

Dong Zhang

Yu-Jiang Zhang

Yuqing Zhang

Sien Zhou

Apostolos Ziakopoulos

Fang Zong

Yajie Zou 